\title{
Editorials
}

\section{Perioperative methemoglobinemia}

\author{
David Johnson MD
}

$\mathrm{T}$

HE failure of oxygen delivery is a subject in which anesthesiologists are well trained. The presence of elevated methemoglobin levels is a consideration that may be overlooked by anesthesiologists despite simple bedside tests that provide clues to the presence of this treatable abnormality. This month's journal cites the clinical experience of one anesthesiologist. ${ }^{1}$ This editorial provides a brief review of methemoglobin.

Methemoglobin is a hemoglobin in which the ferrous $(\mathrm{Fe} 2+)$ irons of heme are oxidized to the ferric $(\mathrm{Fe} 3+)$ state. ${ }^{2}$ The ferric hemes of methemoglobin are unable to bind oxygen and the affinity for oxygen in the remaining ferrous hemes contained within the hemoglobin tetramer is increased. As a result, the oxygen dissociation curve is "left-shifted" and oxygen delivery to the tissues is impaired. In healthy adults, methemoglobin levels do not exceed $1 \%$. Most patients with peak methemoglobin of $8 \%$ or higher are symptomatic. ${ }^{3}$ Methemoglobinemia is due to either increased methemoglobin production or decreased methemoglobin reduction. Auto-oxidation of hemoglobin to methemoglobin occurs spontaneously at a slow rate in normal individuals, converting 0.5 to $3 \%$ of the available hemoglobin to methemoglobin per day. The only physiologically important pathway for reducing methemoglobin back to hemoglobin is the NADPH-dependent reaction catalyzed by cytochrome b5 reductase. An alternative pathway is an enzyme utilizing NADPH generated by glucose-6phosphate dehydrogenase (G6PD) in the hexose monophosphate shunt as a source of electrons. However, there is normally no electron carrier present in red blood cells to interact with NADPH methemoglobin reductase. As a result, electron acceptors such as methylene blue are required for this pathway to work.
Clinical cyanosis occurs when the absolute concentration of methemoglobin exceeds $1.5 \mathrm{~g} \cdot \mathrm{dL}^{-1}$, equivalent to $8-12 \%$ methemoglobin at normal hemoglobin concentrations. Cyanosis from methemoglobinemia cannot be distinguished from other causes of cyanosis. Symptoms (headache, confusion, anxiety, tachypnea) typically occur as methemoglobin increases to 20-30\%. As methemoglobin levels increase further, respiratory depression, altered consciousness, shock, seizures, and death may occur. The presence of symptoms are often lacking in chronic methemoglobinemia because of adaptation to the left shifted hemoglobin. The most common cause of chronic methemoglobinemia is homozygous congenital cytochrome b5 reductase deficiency. A risk factor for acute acquired methemoglobinemia is the asymptomatic heterozygous state for this deficiency. Paradoxically acute toxic methemoglobinemia can be more clinically significant in the heterozygous state compared to the homozygous state because of adaptation in the chronic condition. ${ }^{4}$ Anemic patients may be more sensitive to symptoms of methemoglobinemia because of their lower functional hemoglobin reserve.

Methemoglobinemia should be clinically suspected when "cyanosis" is present with a normal arterial $\mathrm{PO}_{2}$ as obtained by arterial blood gases. The blood in methemoglobinemia is brownish to blue in colour, ${ }^{5}$ and, unlike deoxyhemoglobin, the colour does not change with the addition of oxygen. A quick analysis at the bedside can be done for methemoglobinemia. Normally venous blood exposed to oxygen and shaken turns bright red, while blood containing methemoglobin remains chocolate-brown. ${ }^{5}$ Pulse oximetry is inaccurate in monitoring oxygen saturation in the presence of methemoglobinemia. Methemoglobin absorbs light equally at 660 (oxyhemoglobin) $\mathrm{nm}$ and 940 (deoxyhemoglobin) $\mathrm{nm}$ resulting in a saturation

From the Public Health Sciences, University of Alberta, Edmonton, Alberta, Canada.

Address correspondence to: Dr. David Johnson, Public Health Sciences, University of Alberta, Suite 300, 10216, 124th Street, Edmonton T5N 4E3, Alberta, Canada. Phone: 780-413-5663; E-mail: davidjohnson@cha.ab.ca 
of $85 \%$. An increase in the calculated oxygen saturation gap as cited in this case (arterial blood gas calculated saturation from dissolved oxygen vs the pulse oximetry measured saturation) is another bedside test that should provoke suspicion of methemoglobin. The laboratory diagnosis of methemoglobinemia is based upon analysis of its absorption spectra, which has peak absorbance at $631 \mathrm{~nm}$. A fresh specimen should always be obtained as methemoglobin levels tend to increase with storage. The co-oximeter interprets all readings in the $630 \mathrm{~nm}$ range as methemoglobin; thus, false positives may occur in the presence of other pigments including sulfhemoglobin and methylene blue. Methemoglobin detected by the co-oximeter can be confirmed by chemical assay. ${ }^{6}$

Treatment of methemoglobinemia depends upon the clinical setting (i.e., acute onset of methemoglobinemia due to drugs or other toxic agents ps congenital life-long methemoglobinemia). Offending agents in acquired methemoglobinemia should be discontinued. Methemoglobinemia more than $50 \%$ of total hemoglobin is life-threatening. Blood transfusion or exchange transfusion may be helpful in patients who are in shock. In lesser degrees of methemoglobinemia, no therapy other than discontinuation of the offending agent(s) may be required. If the patient is symptomatic, methylene blue is given intravenously in a dose of 1 to $2 \mathrm{mg} \cdot \mathrm{kg}^{-1}$ over five minutes in order to provide an artificial electron acceptor for the reduction of methemoglobin via the NADPH-dependent pathway. The response is usually rapid and if necessary the dose may be repeated in one hour. Cumulative doses of methylene blue ( $\left.>7 \mathrm{mg} \cdot \mathrm{kg}^{-1}\right)$ can cause dyspnea, chest pain, and hemolysis. Methylene blue is actually an oxidizing agent which accumulates as the production of the reducing agent (leukomethylene blue) becomes saturated. ${ }^{6}$ Serial methemoglobin levels are more reliable when monitoring adequate response to treatment. Repeat methylene blue doses may be necessary as rebound methemoglobinemia up to $12 \mathrm{hr}$ postmethylene blue treatment has been reported due to continued absorption of the inciting drug, metabolite production, or prolonged half-life of the drug. ${ }^{7}$ Since co-oximetry detects methylene blue as methemoglobin, this technique cannot be used to follow the response of methemoglobin levels to treatment with methylene blue. Methylene blue should not be administered to patients with G6PD deficiency, since the reduction of methemoglobin by methylene blue is dependent upon NADPH generated by G6PD. Populations with a high incidence of G6PD deficiency are African-Americans, patients of Mediterranean descent, and Southeast Asians. If methylene blue is contraindicated, ascorbic acid may be given. Hyperbaric oxygen has been used with anecdotal success in severe cases.

The use of oxidizing medications provides the clinical setting for methemoglobin production. The authors in this letter cite a comprehensive list of oxidizing medications. ${ }^{1}$ A number of similar cases have been reported in the literature. Treatment with dapsone $^{8}$ accounted for $42 \%$ of all cases in a retrospective survey of laboratory results. ${ }^{3}$ Methemoglobinemia can be caused by EMLA cream and has been reported with concurrent use of other oxidizing agents such as nitrous oxide. ${ }^{9-11}$ Topical anesthetics such as benzocaine, tetracaine, and butamben mixes or $20 \%$ benzocaine spray can cause methemoglobinemia associated with peak methemoglobin levels of up to $43.8 \%{ }^{3,12,13}$ Diaspirin cross-linked hemoglobin solution is a purified human hemoglobin product which results in an increase of methemoglobin from $0.84 \pm 0.77 \%$ at baseline to $4.08 \pm 1.36 \%{ }^{14}$

In conclusion, methemoglobin as a cause of inadequate oxygen delivery should be considered in the differential diagnosis whenever a paradoxically blue patient with dark blood is noted to have a normal measured $\mathrm{PaO}_{2}$.

\section{La méthémoglobinémie périopératoire}

La défaillance de distribution d'oxygène est un sujet pour lequel les anesthésiologistes sont bien formés. Cependant, la présence de niveaux élevés de méthémoglobine peut être négligée malgré des tests cliniques simples qui fournissent des indices de la présence de cette anomalie traitable. Le présent numéro du journal cite l'expérience clinique d'un anesthésiologiste. ${ }^{1}$ Notre éditorial présente une brève revue sur la méthémoglobine.

La méthémoglobine est une hémoglobine dans laquelle le fer ferreux $(\mathrm{Fe} 2+)$ des hèmes est oxydé en fer ferrique $(\mathrm{Fe} 3+){ }^{2}$ Les hèmes ferriques ne peuvent se lier à l'oxygène et l'affinité pour l'oxygène dans les hèmes ferreux restants, contenus dans le tétramère d'hémoglobine, est augmentée. En conséquence, la courbe de dissociation de l'oxygène est «déviée vers la gauche» et la distribution d'oxygène aux tissus est altérée. Chez les adultes sains, les niveaux de méthémoglobine ne dépassent pas 1 \%. La majorité des 
patients qui ont un taux maximal de $8 \%$ ou plus sont symptomatiques. ${ }^{3}$ La méthémoglobinémie est causée soit par une augmentation de la production de méthémoglobine, soit par une baisse de la réduction de méthémoglobine. L'auto-oxydation de l'hémoglobine en méthémoglobine survient spontanément à un rythme lent chez les individus normaux, convertissant chaque jour de 0,5 à $3 \%$ de l'hémoglobine disponible en méthémoglobine. Le seul mécanisme physiologique important de réduction de la méthémoglobine en hémoglobine est la réaction qui dépend de la NADPH catalysée par la cytochrome b5 réductase. Un autre mécanisme est un enzyme utilisant la NADPH générée par la glucose-6-phosphate déshydrogénase (G6PD) dans la voie des pentoses phosphates comme source d'électrons. Mais, normalement, il n'y a pas de porteur d'électrons dans les globules rouges pour interagir avec la NADPH méthémoglobine réductase. Donc, il faut des accepteurs d'électrons comme le bleu de méthylène pour que ce mécanisme fonctionne.

La cyanose clinique survient quand la concentration absolue de méthémoglobine dépasse $1,5 \mathrm{~g} \cdot \mathrm{dL}^{-1}$, équivalent à 8 à $12 \%$ de méthémoglobine à des concentrations normales d'hémoglobine. La cyanose causée par la méthémoglobinémie ne peut être distinguée de celle qui a d'autres causes. Les symptômes (céphalée, confusion, anxiété, tachypnée) apparaissent généralement quand la méthémoglobine s'élève à 20 à $30 \%$. Quand elle s'élève davantage, une dépression respiratoire, une altération de la conscience, un choc, des convulsions et la mort peuvent survenir. La méthémoglobinémie chronique est rarement symptomatique à cause de l'adaptation à la déviation gauche de la courbe de dissociation de l'hémoglobine. La cause la plus fréquente de méthémoglobinémie chronique est la déficience homozygote congénitale de la cytochrome b5 réductase. Un facteur de risque de méthémoglobinémie aiguë acquise est l'état hétérozygote asymptomatique de cette déficience. Paradoxalement, la méthémoglobinémie toxique aiguë peut être cliniquement plus significative dans l'état hétérozygote, comparé à l'état homozygote, à cause de l'adaptation qui existe dans la chronicité. ${ }^{4}$ Les patients anémiques peuvent être plus sensibles aux symptômes de la méthémoglobinémie, étant donné leur réserve plus faible d'hémoglobine fonctionnelle.

La présence de «cyanose» et d'une $\mathrm{PO}_{2}$ artérielle normale, montrée par la gazométrie du sang artériel, devrait faire penser à la méthémoglobinémie. Le sang est alors de brunâtre à bleu ${ }^{5}$ et, contrairement à la désoxyhémoglobine, sa couleur ne change pas avec l'addition d'oxygène. Une rapide analyse de la méthé- moglobinémie peut être faite au chevet du malade. Le sang veineux, normalement exposé à l'oxygène, puis agité, devient d'un rouge brillant tandis qu'avec la méthémoglobine, il demeure brun chocolat. ${ }^{5} \mathrm{La}$ sphygmo-oxymétrie ne mesure pas efficacement la saturation en oxygène en présence de méthémoglobinémie. La méthémoglobine absorbe la lumière de la même façon à 660 (oxyhémoglobine) nm et à 940 (désoxyhémoglobine) nm, ce qui donne lieu à une saturation de $85 \%$. Une augmentation de l'écart de saturation en oxygène comme dans le cas cité (saturation calculée des gaz du sang artériel à partir de l'oxygène dissous vs saturation mesurée par sphygmooxymétrie) est un autre test de chevet qui devrait alerter sur la présence de méthémoglobine. Le diagnostic expérimental de méthémoglobinémie est fondé sur l'analyse de son spectre d'absorption d'une absorbance maximale à $631 \mathrm{~nm}$. Un échantillon frais devrait toujours être obtenu, les niveaux de méthémoglobine ayant tendance à augmenter avec la conservation. Le co-oxymètre interprète toutes les lectures autour de $630 \mathrm{~nm}$ comme étant de la méthémoglobine ; il peut donc y avoir des faux positifs en présence d'autres pigments, dont la sulfhémoglobine et le bleu de méthylène. On peut confirmer, par analyse chimique, la méthémoglobine détectée par le cooxymètre. $^{6}$

Le traitement de la méthémoglobinémie dépend du tableau clinique (survenue brutale causée par des médicaments ou d'autres agents toxiques vs méthémoglobinémie congénitale permanente). Les agents causals de la méthémoglobinémie acquise doivent être supprimés. La méthémoglobinémie de plus de $50 \%$ de l'hémoglobine totale est une affection grave. La transfusion sanguine ou la transfusion d'échange peut être utile chez des patients en choc. Pour la méthémoglobinémie de moindre degré, le retrait des agents causals suffit. Chez un patient symptomatique, on administre du bleu de méthylène par voie intraveineuse en dose de 1 à $2 \mathrm{mg} \cdot \mathrm{kg}^{-1}$ pendant cinq minutes pour fournir un accepteur d'électrons artificiel en vue de réduire la méthémoglobine par le mécanisme dépendant de la NADPH. La réponse est habituellement rapide et la dose peut être répétée après une heure au besoin. Des doses cumulatives de bleu de méthylène $\left(>7 \mathrm{mg} \cdot \mathrm{kg}^{-1}\right)$ peuvent causer de la dyspnée, de la douleur thoracique et de l'hémolyse. Le bleu de méthylène est, en fait, un agent oxydant qui s'accumule à mesure que la production d'agent réducteur (bleu de leukométhylène) devient saturée. ${ }^{6}$ Les niveaux sériés de méthémoglobine sont mieux évalués avec un monitorage adéquat de la réponse au traitement. Il faudra peut-être répéter les doses de bleu de méthylène, car un rebond de la 
méthémoglobinémie peut se produire jusqu'à $12 \mathrm{~h}$ après le traitement à cause de l'absorption continue du médicament causal, de la production de métabolite ou de la demi-vie prolongée du médicament. ${ }^{7}$ Comme la co-oxymétrie assimile le bleu de méthylène à la méthémoglobine, cette technique ne peut servir pour suivre la réponse au traitement avec le bleu de méthylène. Ce dernier ne devrait pas être administré aux patients qui ont une déficience en G6PD, car la réduction de la méthémoglobine par le bleu de méthylène dépend de la NADPH générée par G6PD. Les populations les plus à risque de déficience en G6PD sont les AfricainsAméricains, les descendants de Méditerranéens et les Sud-Asiatiques. On peut alors administrer de l'acide ascorbique. L'oxygène hyperbare a été utilisé avec un succès empirique dans des cas sévères.

L'usage de médicaments oxydants fournit le cadre clinique à la production de méthémoglobine. Les auteurs de l'article présentent une liste détaillée de médicaments oxydants. ${ }^{1}$ Un certain nombre de cas similaires ont déjà été publiés. Le traitement avec le dapsone $^{8}$ rend compte de $42 \%$ de tous les cas dans une enquête rétrospective des résultats de laboratoire. ${ }^{3}$ La méthémoglobinémie peut être causée par la crème EMLA et a été rapportée avec l'usage concurrent d'autres agents oxydants comme le protoxyde d'azote. ${ }^{9-11}$ Les anesthésiques locaux comme la benzocaïne, la tétracaïne et les mélanges butoformes ou une vaporisation de benzocaïne à $20 \%$ peuvent causer de la méthémoglobinémie associée à des niveaux maximaux de méthémoglobine jusqu'à $43,8 \% .^{3,12,13} \mathrm{La}$ solution croisée d'hémoglobine-diaspirine est un produit d'hémoglobine humaine purifiée qui résulte d'une augmentation de méthémoglobine de 0,84 \pm $0,77 \%$, au départ, à $4,08 \pm 1,36 \% .{ }^{14}$

Finalement, la méthémoglobine comme cause d'un problème de distribution d'oxygène peut être considérée dans le diagnostic différentiel chaque fois qu'un patient présente une cyanose paradoxale, un sang foncé et une $\mathrm{PaO}_{2}$ mesurée normale.

\section{References}

1 Noyes CD, Olufolabi AJ, Habib AS. Subtle desaturation and perioperative methemoglobinemia. The need for continued vigilance (Letter). Can J Anesth 2005; 52: 771-2.

2 Mansouri A, Lurie AA. Concise review: methemoglobinemia. Am J Hematol 1993; 42: 7-12.

3 Ash-Bernal R, Wise R, Wright SM. Acquired methemoglobinemia: a retrospective series of 138 cases at 2 teaching hospitals. Medicine 2004; 83: 265-73.

4 Cohen RJ, Sachs JR, Wicker DJ, Conrad ME. Methemoglobinemia provoked by malarial chemopro- phylaxis in Vietnam. N Engl J Med 1968; 279: 1127-33.

5 Henretig FM, Gribetz B, Kearney T, Lacouture P, Lovejoy $F H$. Interpretation of color change in blood with varying degree of methemoglobinemia. J Toxicol Clin Toxicol 1988; 26: 293-316.

6 Wright RO, Lewander WJ, Woolf AD. Methemoglobinemia: etiology, pharmacology, and clinical management. Ann Emerg Med 1999; 34: 646-56

7 Rehman HU. Methemoglobinemia. West J Med 2001; 175: 193-6.

8 Coleman MD, Rhodes LE, Scott AK, et al. The use of cimetidine to reduce dapsone-dependent methaemoglobinaemia in dermatitis herpetiformis patients. Br J Clin Pharmacol 1992; 34: 244-9.

9 Elsner P, Dummer R. Signs of methaemoglobinaemia after topical application of EMLA cream in an infant with haemangioma. Dermatology 1997; 195: 153-4.

10 Sinisterra S, Miravet E, Alfonso I, Soliz A, Papazian O. Methemoglobinemia in an infant receiving nitric oxide after the use of eutectic mixture of local anesthetic. J Pediatr 2002; 141: 285-6.

11 Hermon MM, Burda G, Golej J, et al. Methemoglobin formation in children with congenital heart disease treated with inhaled nitric oxide after cardiac surgery. Intensive Care Med 2003; 29: 447-52.

12 Jakobson B, Nilsson A. Methemoglobinemia associated with a prilocaine-lidocaine cream and trimetoprim-sulphamethoxazole. A case report. Acta Anaesthesiol Scand $1985 ; 29$ : 453-5.

13 Khan NA, Kruse JA. Methemoglobinemia induced by topical anesthesia: a case report and review. Am J Med Sci 1999; 318: 415-8.

14 O'Hara JF Jr, Colburn WA, Tetzlaff JE, Novick AC, Angermeier KW, Schubert A. Hemoglobin and methemoglobin concentrations after large-dose infusions of diaspirin cross-linked hemoglobin. Anesth Analg 2001; 92: 44-8. 